\title{
Can Dust Destabilise Galactic Disks?
}

\author{
Christian Theis ${ }^{1,3,4}$ and Natalya Orlova ${ }^{2}$ \\ ${ }^{1}$ Institut für Theoretische Physik und Astrophysik der Universität Kiel, 24098 Kiel, Germany \\ ${ }^{2}$ Institute of Physics, Stachki 194, Rostov-on-Don, Russia \\ ${ }^{3}$ Current address: Institut für Astronomie der Universität Wien, 1180 Wien, Austria \\ ${ }^{4}$ E-mail: theis@astro.univie.ac.at
}

Received 2003 October 17, accepted 2004 March 19

\begin{abstract}
We studied the dynamic influence of a dust component on the gaseous phase in central regions of galactic disks. We performed two-dimensional hydrodynamic simulations for flat, multicomponent disks embedded in a stellar and dark matter potential. The pressure-free dust component is coupled to the gas by a drag force depending on their velocity difference. The most unstable regions are those with either a low or near-to-minimum Toomre parameter or with rigid rotation, i.e. the central area. In those regions the dust-free disks become most unstable for a small range of high azimuthal modes $(m \sim 8)$, whereas in dusty disks all modes have similar amplitudes resulting in a patchy appearance. The structures in the dust have a larger contrast between arm and interarm regions than those of the gas. The dust peaks are frequently correlated with peaks of the gas distribution, but they do not necessarily coincide with them. This leads to a large scatter in the dust to gas ratios. The appearance of the dust is more cellular (i.e. sometimes connecting different spiral features), whereas the gas is organised in a multi-armed spiral structure. We found that an admixture of $2 \%$ dust (relative to the mass of the gas) destabilises gaseous disks substantially, whereas dust to gas ratios below $1 \%$ have no influence on the evolution of the gaseous disk. For a high dust to gas ratio of $10 \%$ the instabilities reach the saturation level after $30 \mathrm{Myr}$.
\end{abstract}

Keywords: galaxies: kinematics and dynamics — galaxies: spiral — dust, extinction — ISM: structure

\section{Introduction}

Chemodynamic simulations are characterised by a multiphase treatment of the interstellar medium (ISM). In most of these calculations the ISM is split into two dynamically different components: the clumpy star-forming molecular clouds and the diffuse warm or hot gas (Theis et al. 1992; Samland et al. 1997). More refined decompositions of the ISM are only done for one-zone models (e.g. Ikeuchi et al. 1984), but not for gravitationally coupled, dynamically evolving systems. In particular, the dust component in galaxies is usually just seen as a tracer population which has no influence on galactic dynamics. On the other hand, it is well known that even a small admixture of a dynamically cold component can substantially destabilise galactic structures as Jog \& Solomon (1984) demonstrated for a small amount of cold gas embedded in stellar disks. The aim of our paper is to study whether a dynamically cold dust phase can also destabilise galactic disks.

Good places for looking for the influence of dust are galactic central regions. During recent years, high resolution observations revealed that the circumnuclear regions contain stellar-gaseous mini-disks with sizes of a few hundred pc (e.g. Carollo et al. 1997). These disks have an astonishingly rich structure described as mini-bars, spiral-like dust lanes, star-forming rings, or spiral arms. Many nuclear regions are also well described as patchy or multi-armed. Investigations by Regan \& Mulchaey
(1999) showed that these spirals are the most common morphological structures in the central regions of galaxies.

Since the nature of the mini-spirals is of great importance for our understanding of a variety of astrophysical processes, such as mass accretion onto galactic nuclei, several explanations for them have been invoked. For example, Athanassoula (1992) studied the gas flows in and around bars by (stellar dynamic) orbital analysis. She found that the existence of different periodic orbit families (or equivalently the existence of inner Lindblad resonances, ILRs) is a key criterion for the existence and the shape of dust lanes.

In contrast to the stellar-dynamic interpretation, Englmaier \& Shlosman (2000) suggested that mini-spirals in central regions of galactic disks are related to the formation of grand design spiral patterns in galaxies. They argued that gas density waves - different to stellar density waves - are not completely damped or absorbed at the ILRs and, thus, they may generate spiral structures at all radii including the nuclear regions. Such a model might explain the continuity of some spiral features at small and large radii as well as the low arm-interarm contrast observed in galactic centres. A similar idea of induced structure formation is to invoke secondary bars or small nuclear bars located inside the ILR (Wada \& Koda 2001).

A common property of all the mechanisms mentioned is that they result in structures dominated by two or a few 
arms. On the other hand, Elmegreen et al. (2002) stressed that nuclear dust spirals differ from main-disk spirals in several respects: the nuclear spirals are very irregular with both trailing and leading components that often cross.

All mechanisms discussed have some weak points and cannot fully account for all the observational data. We consider here a new approach based on the observation that circumnuclear disks of galaxies are dusty. By means of a stability analysis Noh et al. (1991) showed that a dust component can strongly destabilise protoplanetary disks. The admixture of only $2 \%$ of dust enhances the growth rates of the dominant gaseous phase significantly. A conservative estimate of the dust to gas ratio $r$ in the solar neighborhood gives an average value of $0.6 \%$, ranging from $0.2 \%$ up to $4 \%$ in $\mathrm{H}_{2}$ regions (Spitzer 1978). These are lower limits because large, massive dust grains do not have a detectable extinction. Direct satellite-based measurements give $r$ values up to $2 \%$ (Frisch et al. 1999). Little is yet known about the true dust to gas ratio in external galaxies, but some galaxies show large values; for example, M51 has a dust to gas ratio of $2 \%$. Taking the observed relation between the dust to gas ratio and the metallicity (Issa et al. 1990) into account, it is reasonable to assume larger dust mass fractions in the metal-enriched central regions of galaxies than on average.

In this paper we study the dynamic influence of a dust component on circumnuclear gaseous disks. We restrict our analysis to systems without a nuclear or large scale bar, and we do not consider any formation or destruction processes of the dust. This investigation is done by twodimensional multicomponent hydrodynamic simulations.

\section{Methods}

\subsection{Equations of Motion}

We studied numerically the hydrodynamic equations for a 2D single or multicomponent disk. Thus, we solved the continuity equation

$$
\frac{\partial \Sigma_{\mathrm{g}, \mathrm{d}}}{\partial t}+\nabla \cdot\left(\Sigma_{\mathrm{g}, \mathrm{d}} \vec{v}_{\mathrm{g}, \mathrm{d}}\right)=0
$$

and the momentum equations for gas and dust. The momentum equations read for gas:

$$
\frac{\partial \vec{v}_{\mathrm{g}}}{\partial t}+\left(\vec{v}_{\mathrm{g}} \cdot \nabla\right) \vec{v}_{\mathrm{g}}+\frac{\nabla P_{\mathrm{g}}}{\Sigma_{\mathrm{g}}}+\nabla\left(\Phi+\Phi_{\mathrm{HBSD}}\right)=S_{\mathrm{g}}\left(\vec{v}_{\mathrm{g}}\right)
$$

and for dust:

$$
\frac{\partial \vec{v}_{\mathrm{d}}}{\partial t}+\left(\vec{v}_{\mathrm{d}} \cdot \nabla\right) \vec{v}_{\mathrm{d}}+\nabla\left(\Phi+\Phi_{\mathrm{HBSD}}\right)=S_{\mathrm{d}}\left(\vec{v}_{\mathrm{d}}\right) .
$$

The components are denoted by $\mathrm{g}$ for the gaseous phase and by $\mathrm{d}$ for the dust. $\Sigma_{\mathrm{g}, \mathrm{d}}$ are the surface densities and $\vec{v}_{\mathrm{g}, \mathrm{d}}$ the velocities. $P_{\mathrm{g}}$ is the pressure of the gas which is given in the 2D case as the force per unit length. $\Phi$ denotes the potential of the self-gravitating disk; it is derived from the Poisson equation

$$
\Delta \Phi=4 \pi G \Sigma(R, \varphi) \delta(z)=4 \pi G\left(\Sigma_{\mathrm{g}}+\Sigma_{\mathrm{d}}\right) \delta(z) .
$$

An external potential is added by a stationary contribution $\Phi_{\text {HBSD }}$ related to the halo, the bulge, and/or a stellar disk component. These external potentials are chosen to match — together with the potential of the disk — a given rotation curve.

The main difference between the gaseous and the dust component is the treatment of the dust as a pressureless phase. Therefore, if gas and dust are in rotational equilibrium, there is a velocity difference between both components which might give rise to a non-negligible frictional force depending on the cross-section for the gas-dust interaction. This interaction is described by the source terms $S(\ldots)$ on the right-hand side of the hydrodynamic equations. Since we do not consider dust formation and destruction processes, the source terms in the continuity equation vanish. However, frictional terms show up in the equations of motion. The dust implementation will be described in the next paragraph.

The set of hydrodynamic equations is closed by a polytropic equation of state

$$
P_{\mathrm{g}}=K \Sigma_{\mathrm{g}}^{\gamma_{\mathrm{g}}}
$$

For the gaseous phase we set the polytropic exponent to $\gamma_{\mathrm{g}}=5 / 3$. The constant $K$ is chosen to yield a given minimum Toomre parameter.

\subsection{Treatment of the Dust Component}

The main difference between 'normal' galactic disks and our dusty disks is that the cold component (dust) is not only coupled by gravity to the hotter phase (gas), but also by a frictional force between both components.

This drag is taken into account by a source term in the equations of motion following the general form suggested by Noh et al. (1991):

$$
\begin{gathered}
\vec{f} \equiv S_{\mathrm{d}}\left(\vec{v}_{\mathrm{d}}\right)=-A\left(\vec{v}_{\mathrm{d}}-\vec{v}_{\mathrm{g}}\right) \\
S_{\mathrm{g}}\left(\vec{v}_{\mathrm{g}}\right)=-\frac{\Sigma_{\mathrm{d}}}{\Sigma_{\mathrm{g}}} \vec{f} .
\end{gathered}
$$

The second source term, Equation (7), follows from the requirement of momentum conservation. The physics of the friction is enclosed in the frictional timescale $A^{-1}$. Based on a microscopic view of the frictional process, i.e. the momentum exchange between gas and dust particles by collisions and the equipartition of momentum within the gaseous phase, the timescales can be calculated. For the simulations shown in this paper we assumed that the dust disk is thin compared to the gaseous disk. In that case the timescale of the friction is of the order of the timescale of the dynamics given by the inverse circular frequency $\Omega^{-1}$ (Noh et al. 1991), i.e.

$$
A=\Omega \text {. }
$$

The basic assumption is here that gas and dust establish very fast collisional equilibrium in the thin dust layer and that the gas momentum is then mixed vertically in a soundtravelling timescale (for further details see Theis \& Orlova 2004). 

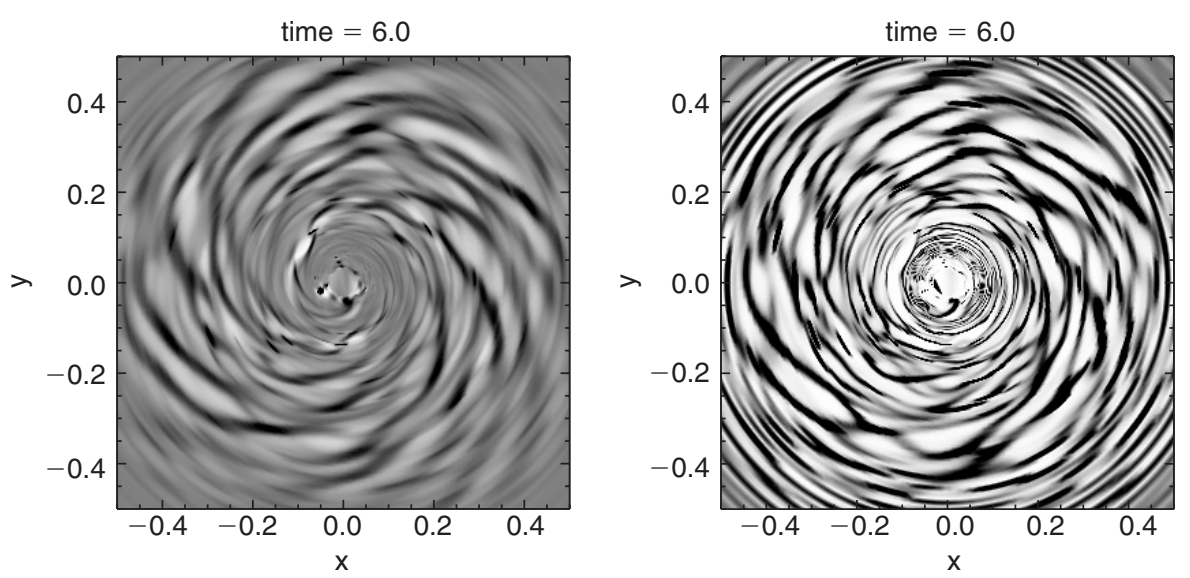

Figure 1 Perturbations of the surface density (normalised to their initial values) of the gas and dust components after $t=6 \sim 90$ Myr for a model with a $10 \%$ dust mass fraction (relative to gas): $\Delta \Sigma_{\mathrm{g}, \mathrm{d}} / \Sigma_{\mathrm{g}, \mathrm{d}}$ for gas (left) and dust (right). Areas devoid of material are white; areas with a density enhancement of a factor of two or more are black. The grey areas seen at the outer edges correspond to no deviation from the initial surface density. The lengths at the $x$ and $y$ axes are given in kpc.

\subsection{Numerical Implementation}

The nonlinear analysis implies the solution of the full set of hydrodynamic equations. For this purpose, we developed a 2D numeric code which is similar to the ZEUS-2D code by Stone \& Norman (1992). The hydrodynamic equations are made discrete on a logarithmic Eulerian grid in polar coordinates $(270 \times 270$ grid cells $)$. The different terms are treated by operator splitting. Advection is performed by a second order van Leer advection scheme.

\section{Results}

\subsection{Parameters of the Initial Gaseous Disk}

The characteristic values of our initial models are motivated by the nuclear region of M100 (NGC 4321). We adopted a total gas mass of $4.7 \times 10^{8} \mathrm{M}_{\odot}$ distributed exponentially with a scale length of $300 \mathrm{pc}$ within a radial range of $R_{\text {in }}=30 \mathrm{pc}$ to $R_{\text {out }}=3 \mathrm{kpc}$. The short disk scale length mimics a central concentration of (molecular) gas observed in many galaxies. Small random perturbations are initially superimposed on the mass distribution by multiplying the surface density in each cell by a factor $\left(1+A_{R} R\right) . R$ is a random number in the interval $[-1,1]$ and $A_{R}$ a small amplitude of the order of $10^{-8}$.

The initial velocities are derived from rotational equilibrium, i.e. there is no initial radial motion. The rotation curve is specified by

$$
v_{c}(R)=v_{\infty} \cdot \frac{R / R_{\text {flat }}}{\left[1+\left(R / R_{\text {flat }}\right)^{n_{t}}\right]^{1 / n_{t}}} .
$$

The velocity at infinity, $v_{\infty}$, was set to $178 \mathrm{~km} \mathrm{~s}^{-1}$. The transition parameter $n_{t}$ was selected to be 10 , resulting in a fairly sharp transition at the radius $R_{\text {flat }}=100 \mathrm{pc}$. This rotation curve corresponds to a total dynamic mass $M_{\mathrm{d}}(R) \sim v_{c}^{2}(R) R / G$ (including all components) of $7.3 \times 10^{8} \mathrm{M}_{\odot}$ within the central $100 \mathrm{pc}$. In the region of rigid rotation the rotation period is about $3.5 \times 10^{6} \mathrm{yr}$. It increases outwards reaching $1.7 \times 10^{7} \mathrm{yr}$ at the halfmass radius of the gaseous component at $R \approx 500 \mathrm{pc}$. The azimuthal velocity of the gaseous phase is calculated by the (frictionless) Jeans' equation.

According to the chosen rotation curve, mass profile, and equation of state (polytropic with $\gamma_{\mathrm{g}}=5 / 3$ ), the minimum value of the Toomre parameter is reached at a galactocentric distance of about $440 \mathrm{pc}$. The constant in the equation of state (for the gaseous phase) was selected to yield a minimum Toomre parameter of $Q_{\min }=1.54$. This choice corresponds to sound speeds between 4 and $11 \mathrm{~km} \mathrm{~s}^{-1}$ within the central $\mathrm{kpc}$ (the higher value is reached in the centre).

\subsection{A Dusty Disk}

We studied the influence of the dust to gas mass ratio $r$ by varying $r \equiv M_{\mathrm{d}} / M_{\mathrm{g}}$ from $0.5 \%$ to $20 \%$.

Figure 1 shows the surface density perturbations of gas and dust in the saturation stage of a model with a large dust to gas ratio: the irregular multi-armed structure is emphasised due to large arm-interarm variations. Along the arms strong surface density variations can be discerned. Some arms are interrupted by low density areas; others are not smoothly curved, but show wiggles. Some arms seem to merge with others. The dust distribution is highly correlated with the gas distribution. However, the contrast between arm and interarm regions is larger for the dust than for the gas. The surface densities of the dust vary by about one order of magnitude, whereas the contrast of the gas component is usually less than a factor of two. The structures formed in the dust are also thinner than those of the gas. Though there is a tight correlation between the positions of the maxima of the gas and dust phases, there is no correlation between their maximum amplitudes. The dust distribution is characterised by a more cellular appearance compared to the spiral-like morphology of the gas.

It is interesting to note that the dust is often located at the boundaries around peaks of the gas distribution, preferentially at the inner boundary. Other dust peaks are in regions with no, or only weak, gaseous density enhancements. And, of course, there are dust peaks at the 


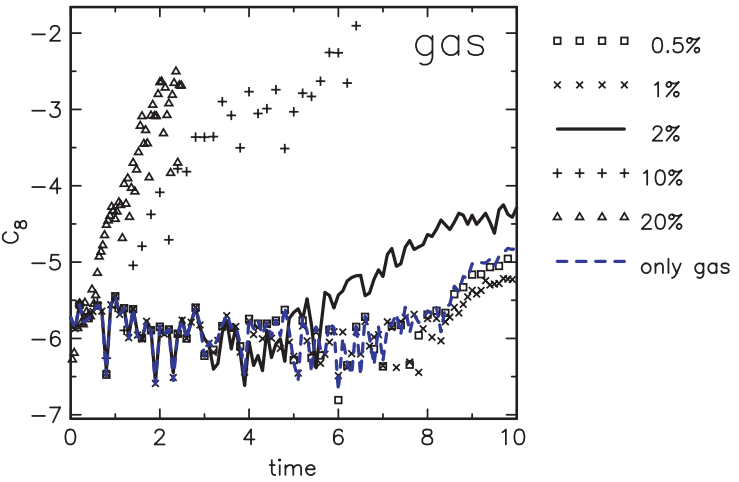

Figure 2 Temporal evolution of the logarithmic Fourier amplitudes $C_{8} \equiv \log A_{8}$ of the (dominant) $m=8$ mode of the dust component for different dust to gas mass fractions: $0.5 \%$ (open boxes), $1 \%$ (crosses), 2\% (reference model, solid line), 10\% (plus), 20\% (triangle), and the purely gaseous model (dashed line). The time unit is $1.5 \times 10^{7} \mathrm{yr}$.

same locations as those of the gas. Thus, one expects large spatial variations of the dust to gas ratio.

In order to quantify the (global) stability of the disk we use the global Fourier amplitudes of each component:

$A_{m} \equiv \frac{1}{M_{\text {disk }}}\left|\int_{0}^{2 \pi} \int_{R_{\text {in }}}^{R_{\text {out }}} \Sigma(r, \phi) r \mathrm{~d} r \mathrm{e}^{-\mathrm{i} m \phi} \mathrm{d} \phi\right| \quad(m>0)$.

$M_{\text {disk }}$ is the mass of the disk for the component of interest in the specified radial interval $\left[R_{\text {in }}, R_{\text {out }}\right]$. $\Sigma$ denotes the corresponding surface density. Comparing the modes from $m=1$ to $m=16$, the high $m$ modes around $m \sim 8$ are dominant during the linear regime. A comparison of the corresponding Fourier amplitudes with those of a purely gaseous model shows that the critical dust to gas ratio $r_{c}$ for dust becoming dynamically unimportant is about $1 \%$ (Figure 2). For larger amounts of dust the destabilisation of the gaseous phase becomes much stronger. For $r=0.02$ the instability sets in about $50 \mathrm{Myr}$ earlier than in dustless or low dust-content models, while the modes remain on their initial value for a latency period of about $75 \mathrm{Myr}$. Increasing $r$ to $10 \%$ reduces the latency time to almost zero. The growth rates increase by a factor of 3-4 and the saturation level reached after $30 \mathrm{Myr}$ is larger by at least one order of magnitude.

A more detailed discussion on the method and the results as well as an extended parameter study can be found in our succeeding paper (Theis \& Orlova 2004).

\section{Summary}

We studied the influence of a cold dust component on the evolution of galactic gaseous disks by means of two-dimensional hydrodynamic simulations. From the evolution of the Fourier amplitudes we found that the higher order modes are the dominant unstable modes. Their growth is mainly restricted to the central kiloparsec. An admixture of $2 \%$ dust (relative to the gas mass) destabilises the gaseous disk. The growth of instabilities in the dust component becomes non-linear after $100 \mathrm{Myr}$, followed by the gas $50 \mathrm{Myr}$ later.
The structures formed in both gas and dust are rather irregular and multi-armed. This is a direct consequence of the superposition of many high $m$ modes of similar amplitude. The dust component is characterised by a much stronger contrast between arm and interarm regions than the gas. The dust is spatially correlated with gas, but it does not exactly follow the gas. Peaks in the dust distribution are frequently found at the inner edges of peaks in the gas distribution. This results in a large scatter of dust to gas ratios at different places. The dust also develops thin filaments which sometimes connect the arms. Therefore, the dust distribution has a more cellular appearance, whereas the gas develops a multi-armed spiral morphology.

Below a dust to gas mass ratio of $1 \%$ the dynamic influence of the dust on the gaseous disk becomes negligible. This critical value is close to the observed mean value in normal galaxies like the Milky Way. Since the dust to gas ratio scales linearily with metallicity, larger local values of $r$, especially in the central galactic regions, seem to be reasonable. Such values are also in agreement with local gas to dust determinations. Since a dust to gas ratio of $2 \%$ significantly affects the evolution of the disk, even the observed small dust admixtures are expected to have an impact on the dynamics of some galaxies (e.g. the dust-rich M51). For a 10\% admixture of dust the gaseous component is completely destabilised. The growth rates are enhanced by a factor of 3-4 with almost no latency phase. The saturation levels reached after $30 \mathrm{Myr}$ are substantially larger than in the low $r$ calculations.

\section{Acknowledgements}

The authors thank Vladimir Korchagin for stimulating discussions about disk stability. This work has been supported by the DFG under grants TH511/2-3 and RUS 436 RUS $17 / 65 / 02$. C. T. is also grateful to the organisers of the GCD-V meeting for a very enjoyable conference as well as for financial support making his participation possible. The simulations were performed at the computing center of the Christian-Albrechts-Universität Kiel.

\section{References}

Athanassoula, E. 1992, MNRAS, 259, 345

Carollo, C. M., Stiavelli, M., de Zeeuw, P. T., \& Mack, J. 1997, AJ, 114,2366

Elmegreen, B. G., Elmegreen, D. M., \& Eberwein, K. S. 2002, ApJ, 564,234

Englmaier, P., \& Shlosman, I. 2000, ApJ, 528, 677

Frisch, P. C., et al. 1999, ApJ, 525, 492

Ikeuchi, S., Habe, A., \& Tanaka, Y. D. 1984, MNRAS, 207, 909

Issa, M. R., MacLaren, I., \& Wolfendale, A. W. 1990, A\&A, 236, 237

Jog, C. J., \& Solomon, P. M. 1984, ApJ, 276, 114

Noh, H., Vishniac, E. T., \& Cochran, W. 1991, ApJ, 383, 372

Regan, M. W., \& Mulchaey, J. S. 1999, AJ, 117, 2676

Samland, M., Hensler, G., \& Theis, Ch. 1997, ApJ, 476, 544

Spitzer, L. 1978, Physical Processes in the Interstellar Medium (New York: Wiley)

Stone, J. M., \& Norman, M. L. 1992, ApJS, 80, 753

Theis, Ch., \& Orlova, N. 2004, A\&A, 418, 959

Theis, Ch., Burkert, A., \& Hensler, G. 1992, A\&A, 265, 465

Wada, K., \& Koda, J. 2001, PASJ, 53, 1163 